\title{
Les hydroxyéthylamidons, 20 ans après
}

\section{The hydroxyethylstarchs, 20 years later}

\author{
F. Schortgen $\cdot$ C. Richard \\ (C) SRLF et Springer-Verlag France 2013
}

Au cours de l'insuffisance circulatoire, la correction rapide de l'hypotension constitue un des éléments-clés du pronostic [1]. Dans ce contexte, le remplissage vasculaire (RV) occupe, dans la majorité des cas, une place centrale, posant au quotidien le problème du rapport bénéfice-risque de son utilisation. En cherchant systématiquement à privilégier le bénéfice hémodynamique, le développement depuis plus d'un demisiècle puis la commercialisation de macromolécules synthétiques moins chères que l'albumine ont été réalisés afin de mettre à la disposition du réanimateur des solutions de remplissage augmentant, en référence aux cristalloïdes, rapidement et durablement le volume plasmatique [2]. Il s'est successivement agi des dextrans dans les années quarante, des gélatines dans les années cinquante et des hydroxyéthylamidons (HEA) dans les années soixante. C'est plus récemment que s'est développée la culture de l'évaluation du risque thérapeutique, en particulier dans ces situations d'urgence vitale, où la préoccupation légitime du réanimateur est d'assurer la survie immédiate du patient. À court terme, la prévention du risque de surcharge pulmonaire liée au RV a beaucoup bénéficié de l'évaluation de la précharge-dépendance et de la prédiction de l'efficacité du RV à l'aide d'indices hémodynamiques développés en particulier chez le patient sous ventilation mécanique [3]. L'évaluation du rapport bénéfice/risque chez les malades de réanimation a longtemps reposé sur l'analyse de la mortalité à J28. Ce critère de jugement, aujourd'hui insuffisant, est complété par l'analyse de la cinétique d'évolution des défaillances d'organe, en particulier rénal, tant à court que moyen et long termes. L'histoire du développement et de la commercialisation des HEA illustre cette évolution.

C'est en 1991 qu'a été commercialisée en France la première solution d'HEA (Elohes ${ }^{\circledR}$, HEA $200 \mathrm{kDa} / 0,6$ ). L'origine végétale des HEA et leur fort pouvoir d'expansion volémique ont rapidement favorisé leur utilisation aux dépens des

\footnotetext{
F. Schortgen

Service de réanimation médicale, hôpital Henri Mondor (AP-HP)

C. Richard $(\bowtie)$

Service de réanimation médicale, hôpital de Bicêtre (AP-HP), Université Paris Sud 11, EA533

e-mail : christian.richard@bct.aphp.fr
}

gélatines. Dès la première année d'utilisation, une augmentation significative des lésions de néphrose osmotique a été observée chez les transplantés rénaux [4]. Deux essais randomisés réalisés par des équipes françaises, l'un publié en 1996 concernant les donneurs d'organes [5], l'autre en 2001 concernant des patients en sepsis sévère [6], ont confirmé les effets délétères des HEA sur la fonction rénale. L'existence d'un lien entre la présence d'une défaillance rénale et la mortalité chez le patient de réanimation a amené à s'interroger sur l'intérêt de l'utilisation d'un colloïde, ayant certes un fort pouvoir d'expansion volémique, mais dont le risque de toxicité rénale était largement suspecté alors qu'existait, avec les solutés cristalloïdes, une évidente alternative.

La stratégie adoptée par l'industrie pharmaceutique a alors été de diminuer la demi-vie d'élimination des HEA en réduisant leur poids moléculaire et leur pourcentage de substitution en radicaux hydroxylés (HEA de $2^{\mathrm{e}}$ et $3^{\mathrm{e}}$ générations), afin de chercher à en limiter l'accumulation intratissulaire. Un essai randomisé (VISEP), conduit sur patients en sepsis sévère, comparant un HEA de $2^{\mathrm{e}}$ génération (HES 200/0,5) à un cristalloïde, le Ringer lactate, a été prématurément interrompu, après l'inclusion de 537 patients, en raison d'une incidence accrue d'insuffisance rénale dans le groupe HEA alors qu'était observée une normalisation plus précoce de la pression veineuse centrale [7]. Dans cette étude, une augmentation de $20 \%$ de la mortalité relative à $\mathrm{J} 90(\mathrm{p}=0,09)$ dans le groupe HEA a été observée, en lien étroit avec les doses cumulées [7].

En dépit de la confirmation de la toxicité rénale des HEA de $2^{\mathrm{e}}$ génération, une $3^{\mathrm{e}}$ génération de HEA de poids moléculaire et de degré de substitution encore réduits (HEA 130/0,40-0,42) a cependant été commercialisée sur la base d'études montrant une efficacité équivalente comparée aux générations précédentes [8]. L'analyse de la littérature concernant le rapport bénéfice/risque de l'utilisation des HEA de $3^{\mathrm{e}}$ génération a été rendue difficile par la décision du retrait des bases de données de 19 publications du même auteur concernant l'HEA 130/0,4 utilisé au cours de l'anesthésie générale et/ou de la période postopératoire [8].Une méta-analyse publiée au début de l'année 2012 n'avait pas permis de conclure en raison de la médiocrité méthodologique des 
études préalables et confirmait la nécessité d'attendre les résultats des études prospectives randomisées en cours. L'objectif principal de ces études était double : la toxicité rénale des HEA correspondait-elle à un effet de classe médicamenteuse quels que soient le poids moléculaire et/ou le pourcentage de substitution, et ce risque était-il l'apanage des patients en sepsis sévère ? Dès 2011, dans l'attente des résultats de ces essais randomisés, l'European Society of Intensive Care Medicine (ESICM) avait établi et publié des recommandations préconisant de ne pas utiliser les HEA chez les patients septiques [9].

Les trois essais randomisés publiés en 2012, comparant un HEA de $3^{\mathrm{e}}$ génération à un cristalloïde, ont apporté des réponses importantes à ces deux questions. L'étude CRYSTMAS réalisée sur un collectif de 174 patients hospitalisés pour sepsis sévère, a mis en évidence une incidence plus élevée à $\mathrm{J} 90$ de recours à l'hémodialyse chez les patients recevant de l'HEA (21 vs $11 \%$ ) ainsi qu'une mortalité supérieure ( 40 vs $34 \%$ ), ces résultats étant cependant statistiquement non significatifs probablement en regard de la faible puissance de l'étude [10]. Réalisée chez 798 patients hospitalisés pour sepsis sévère, l'étude $6 \mathrm{~S}$ a confirmé une mortalité significativement augmentée et un recours plus fréquent à l'hémodialyse chez les patients recevant de l'HEA [11]. Enfin, l'étude CHEST réalisée sur une population hétérogène de 7000 patients nécessitant un RV a montré un recours significativement plus important à l'hémodialyse chez les patients recevant de l'HEA et ce en dépit d'un moindre recours aux vasopresseurs [12]. Tant dans l'étude 6S que dans l'étude CHEST, une augmentation significative du recours aux transfusions globulaires et le constat d'une incidence accrue de défaillances hépatiques ont été observés $[11,12]$. Prenant en compte ces résultats, une méta-analyse présentée en octobre 2012 par Gattas et al. (Intensive Care Medicine, in press) au congrès de l'ESICM indiquait que l'expansion volémique réalisée avec un HEA 130/0,40 ou 0,42 augmentait le risque relatif de décès de $8 \%$ (intervalle de confiance-95\%, 0-17) et de recours à l'hémodialyse de $25 \%$ (intervalle de confiance- $95 \%, 8-44$ ).

Le risque de toxicité rénale des HEA apparaît donc commun aux trois générations de solutions et pourrait, au vu des résultats de l'étude CHEST, ne pas être l'apanage des patients de réanimation souffrant de sepsis sévère [12]. Plus de vingt ans auront été nécessaires depuis la première publication concernant la toxicité rénale des HEA, pour que la Food and Drug Administration et l'European Medicines Agency décident d'entamer un processus de réévaluation de leur rapport bénéfice/risque. L'Agence nationale de sécurité du médicament devrait s'emparer de ce sujet.

Au-delà du problème spécifique des HEA, ce dossier éclaire d'un jour nouveau la problématique de l'évaluation du bénéfice/risque d'un médicament destiné à la prise en charge en urgence des patients souffrant d'insuffisance circu- latoire. Cette évaluation, longtemps limitée à celle de la cinétique de la correction des désordres hémodynamiques et en particulier de l'hypotension artérielle, apparaît aujourd'hui largement insuffisante. Le rapport bénéfice/risque doit désormais impérativement faire l'objet d'une évaluation certes à court terme mais aussi à moyen et long termes. L'apparent paradoxe d'une augmentation de l'incidence de la défaillance rénale et du recours à l'hémodialyse associés dans les études concernant les patients atteints de sepsis sévère à une surmortalité amène à s'interroger sur la pertinence des critères de jugement hémodynamiques habituellement utilisés. Ces critères qui, jusqu'à ce jour, ont prévalu pour le développement et la commercialisation des solutions de macromolécules synthétiques, doivent faire l'objet d'une réévaluation. L'utilisation des cristalloïdes dont les rapports bénéfice/risque et coût/efficacité apparaissent plus favorables que ceux des macromolécules synthétiques doit être privilégiée pour la prise en charge d'une insuffisance circulatoire, tout particulièrement dans le contexte d'un sepsis sévère.

\section{Références}

1. Dellinger RP, Levy MM, Carlet JM, et al (2008) Surviving Sepsis Campaign: international guidelines for management of severe sepsis and septic shock: 2008. Crit Care Med 36:296-327

2. Schortgen F, Deye N, Brochard L (2004) Preferred plasma volume expanders for critically ill patients: results of an international survey. Intensive Care Med 30:2222-9

3. Marik PE, Monnet X, Teboul JL (2011) Hemodynamic parameters to guide fluid therapy. Ann Intensive Care 1:1

4. Legendre C, Thervet E, Page B, et al (1993) Hydroxyethylstarch and osmotic-nephrosis-like lesions in kidney transplantation. Lancet 342:248-9

5. Cittanova ML, Leblanc I, Legendre C, et al (1996) Effect of hydroxyethylstarch in brain-dead kidney donors on renal function in kidney-transplant recipients. Lancet 348:1620-2

6. Schortgen F, Lacherade JC, Bruneel F, et al (2001) Effects of hydroxyethylstarch and gelatin on renal function in severe sepsis: a multicentre randomised study. Lancet 357:911-6

7. Brunkhorst FM, Engel C, Bloos F, et al (2008) Intensive insulin therapy and pentastarch resuscitation in severe sepsis. N Engl J Med 358:125-39

8. Gattas DJ, Dan A, Myburgh J, et al (2012) Fluid resuscitation with $6 \%$ hydroxyethyl starch $(130 / 0.4)$ in acutely ill patients: an updated systematic review and meta-analysis. Anesth Analg 114:159-69

9. Reinhart K, Perner A, Sprung CL, et al (2012) Consensus statement of the ESICM task force on colloid volume therapy in critically ill patients. Intensive Care Med 38:368-83

10. Guidet B, Martinet O, Boulain T, et al (2012) Assessment of hemodynamic efficacy and safety of $6 \%$ hydroxyethylstarch $130 / 0.4$ vs. $0.9 \% \mathrm{NaCl}$ fluid replacement in patients with severe sepsis: The CRYSTMAS study. Crit Care 16:R94

11. Perner A, Haase N, Guttormsen AB, et al (2012) Hydroxyethyl starch 130/0.42 versus Ringer's acetate in severe sepsis. N Engl J Med 367:124-34

12. Myburgh JA, Finfer S, Bellomo R, et al (2012) Hydroxyethyl starch or saline for fluid resuscitation in intensive care. N Engl J Med 367:1901-11 